\title{
COVID-19: Una nueva perspectiva en gastroenterología
}

\section{COVID-19: A new perspective in gastroenterology}

Rommel Zambrano-Huailla, MD. ${ }^{1}$

1 Servicio de gastroenterología, Hospital III de Emergencias Grau EsSalud. Lima, Perú.

*Correspondencia: Rommel Zambrano-Huailla, MD rommel_334@hotmail.com

Fecha recibido: $\quad 29 / 04 / 20$ Fecha aceptado: $12 / 05 / 20$
Desde su aparición en Wuhan, provincia de Hubei, en China central, y producto de su rápida propagación y gravedad, el nuevo coronavirus (2019-nCov) ha sido declarado como "emergencia de salud pública de importancia internacional" por la Organización Mundial de la Salud (OMS). Cabe mencionar que es la sexta vez que la OMS realiza un pronunciamiento de tal magnitud desde la aprobación del Reglamento Sanitario Internacional en el 2005 (1).

Esta enfermedad se transmite de persona a persona a través de aerosoles generados por tos o estornudos, que en su etapa más crítica desarrolla el síndrome respiratorio agudo severo (SARS), condición que en un gran porcentaje de pacientes demandará ventilación mecánica. Sin embargo, diversos estudios publicados y en fase de publicación reportan también síntomas gastrointestinales en pacientes infectados, en los que se destaca la presencia de diarrea (Tabla 1) (2-6).

Tabla 1. Manifestaciones gastrointestinales de pacientes con COVID-19 según el país de estudio

\begin{tabular}{|lccccc|}
\multicolumn{1}{c}{ Primer autor } & País & Pacientes & Diarrea & $\begin{array}{c}\text { Náuseal } \\
\text { vómito }\end{array}$ & $\begin{array}{c}\text { Dolor } \\
\text { abdominal }\end{array}$ \\
\hline Majeed y cols. (2) & China & 2748 & $245(9,1 \%)$ & $133(7 \%)$ & $17(2,4 \%)$ \\
\hline Garg y cols. (3) & Estados Unidos & 180 & $48(26,7 \%)$ & $44(24,4 \%)$ & $15(8,3 \%)$ \\
\hline Kim y cols. (4) & Corea del Sur & 101 & $3(2,97 \%)$ & $6(5,94 \%)$ & $3(2,97 \%)$ \\
\hline Shing Cheung y cols. (5) & Hong Kong & 59 & $13(22 \%)$ & $1(1,7 \%)$ & $1(11,9 \%)$ \\
\hline Escalera-Antezan y cols. (6) & Bolivia & 12 & $2(16,6 \%)$ & $1(8,3 \%)$ & $2(16,6 \%)$
\end{tabular}

El 2019-nCov utiliza la enzima convertidora de angiotensina 2 (ECA-2) para ingresar a la célula. Esta no solo se encuentra en el pulmón, también se expresa en células del tracto digestivo, colangiocitos y hepatocitos $(7,8)$. Esto último explicaría las manifestaciones gastrointestinales reportadas y, a su vez, fundamentaría una posible transmisión fecal-oral de la enfermedad (7). Este contexto convierte automáticamente a los centros endoscópicos en lugares de alto riesgo.

En tal sentido, diferentes sociedades han manifestado sus recomendaciones y lineamientos para la atención en áreas de procedimientos endoscópicos, así como también en el seguimiento de pacientes ambulatorios. Entonces, la Asociación Americana de 
Gastroenterología (AGA) recomienda el correcto uso del equipo de protección personal y el empleo de cuartos de presión negativa durante la realización de la endoscopia de un paciente confirmado o sospechoso de COVID-19 (9).

Por su parte, la Asociación Europea para el Estudio del Hígado (EASL) sugiere, en pacientes ambulatorios, reducir la frecuencia de visitas a los establecimientos de salud mediante el uso de la telemedicina, con el fin de disminuir la exposición de pacientes vulnerables al COVID-19 (10). Esto revela que la práctica clínica diaria sufrirá cambios en los meses venideros y dependerá de nuestra capacidad de adaptación para seguir ofreciendo los mismos estándares de atención a nuestros pacientes.
Desde otro punto de vista, es una oportunidad para el desarrollo de líneas de investigación. Es preciso desarrollar estudios con el objetivo de evidenciar las manifestaciones del COVID-19 en el tracto digestivo, así como también evaluar su impacto en pacientes con enfermedades gastrointestinales crónicas. Esto permitirá una mayor comprensión del comportamiento de esta enfermedad en nuestra región.

En conclusión, es difícil determinar el impacto real de esta epidemia; a pesar de ello, se crean nuevas oportunidades para el desarrollo de competencias y herramientas que marcarán el rumbo de la atención de los pacientes.

\section{REFERENCIAS}

1. Ramos C, C D. Covid-19 : la nueva enfermedad causada por un coronavirus. Salud Publica Mex [Internet]. 2020;62(2):225-7.

Available from: https://doi.org/10.21149/11276

2. Majeed MB, Majeed MB, Agrawal R, Wang Y, Attar B, Patel P, et al. Prevalence of gastrointestinal symptoms in COVID-19 patients and impact of medical resources on disease transmission. Available-at SSRN. 3572865(2020). https://doi.org/10.2139/ssrn.3572865

3. Garg S, Kim L, Whitaker M, O'Halloran A, Cummings C, Holstein R, et al. Hospitalization Rates and Characteristics of Patients Hospitalized with Laboratory-Confirmed Coronavirus Disease 2019 - COVID-NET, 14 States, March 1-30, 2020. MMWR Morb Mortal Wkly Rep. 2020;69(15):458-64. https://doi.org/10.15585/mmwr.mm6915e3

4. Kim T, Park O, Ph D, Kim G, Ph D. Infectious Diseases Epidemiological and Clinical Characteristics of Early 101 Deceased Patients in the Coronavirus Disease-19 (COVID19) Outbreak in Republic of Korea. (2020). Available-at SSRN https//ssrn.com/abstract $=3571538$. https://doi.org/10.2139/ssrn.3571538

5. Cheung KS, Hung IF, Chan PP, Lung KC, Tso E, Liu R, et al. Gastrointestinal Manifestations of SARS-CoV-2 Infection and Virus Load in Fecal Samples from the Hong Kong Cohort and Systematic Review and Meta-analysis. Gastroenterology [Internet]. 2020. https://doi.org/10.1053/j.gastro.2020.03.065
6. Escalera-antezana JP, Lizon-ferrufino NF, Maldonadoalanoca A, Alarcón-de-la-vega G, Alvarado-arnez LE, Balderrama-saavedra MA, et al. Clinical features of the first cases and a cluster of Coronavirus Disease 2019 (COVID19) in Bolivia imported from Italy and Spain. Travel Med Infect Dis [Internet]. 2020; (March):101653. Available from: https://doi.org/10.1016/j.tmaid.2020.101653

7. Xiao F, Tang M, Zheng X, Liu Y, Shan H. Evidence for Gastrointestinal Infection of SARS-CoV-2. Gastroenterology. 2020;S0016-5085. https://doi.org/10.1053/j.gastro.2020.02.055

8. Chai X, Hu L, Zhang Y, Han W, Lu Z, Ke A. Specific ACE2 Expression in Cholangiocytes May Cause Liver Damage After 2019-nCoV Infection. bioRxiv [Internet]. 2020;2020.02.03.931766. Available from: http://biorxiv.org/ content/early/2020/02/04/2020.02.03.931766.abstract

9. Sultan S, Lim JK, Altayar O, Davitkov P, Joseph D, Siddique $\mathrm{SM}$, et al. AGA Institute Rapid Recommendations for Gastrointestinal Procedures During the COVID-19 Pandemic. Gastroenterology [Internet]. 2020; Available from: https://doi.org/10.1053/j.gastro.2020.03.072

10. Boettler T, Newsome PN, Mondelli MU, Maticic M, Cordero E. Care of patients with liver disease during the COVID-19 pandemic: EASL-ESCMID Position Paper. JHEP Reports [Internet]. 2020;2(3):100113. Available from: www.sciencedirect.com/science/article/pii/ S2589555920300471 https://doi.org/10.1016/j.jhepr.2020.100113 